\title{
Les marqueurs biologiques de la consommation d'alcool
}

\section{Biological markers of alcohol consumption}

Michel LHERMITTE*(1), André KLEIN ${ }^{(1}$, Thierry DANEL ${ }^{(2)}$

(1) Laboratoire de Biochimie et de Biologie Moléculaire, Hôpital Calmette, CHRU DE Lille, Avenue du Professeur J. Leclercq - 59037 LILLE Cedex

(2) Service d'Addictologie, Clinique de la Charité, 57, boulevard de Metz, CHRU de Lille - 59037 LILLE Cedex

* Auteur à qui adresser la correspondance : Michel LHERMITTE, Laboratoire de Biochimie et de Biologie Moléculaire, Hôpital Calmette, CHRU de Lille, Avenue du Professeur J. Leclercq - 59037 LILLE Cedex Tél : +33 (0)32044 4963 - Fax : +33 (0) 20444729 - e-mail : mlhermitte@chru-lille.fr

(Reçu le $1^{\text {er }}$ février 2002 ; accepté le 15 février 2002)

\section{RÉSUMÉ}

Les problèmes liés à la consommation d'alcool sont parmi les plus importants en addictologie, et constituent un problème majeur en clinique. Plusieurs anomalies biologiques peuvent être remarquées après consommation d'alcool. Certains paramètres biologiques sont importants à prendre en compte, pour faire le diagnostic et lors des traitements des sujets, notamment lors du sevrage des patients. Les différents marqueurs biologiques de l'alcoolisation sont présentés : la gamma-glutamyltransférase, les transaminases, la transferrine deficiente en glycanes, le volume globulaire moyen, et un nouveau paramètre, la $\gamma$-CDT. Aucun test, à lui seul, n'est assez sensible, ni spécifique pour faire le diagnostic. La combinaison de l'anamnèse, des questionnaires et des marqueurs biologiques est nécessaire pour évoquer le diagnostic de troubles liés à l'alcool.

\section{MOTS-CLÉS}

Alcoolisme, gamma-glutamyltransférase, transferrine déficiente en hydrates de carbone (CDT), $\gamma$-CDT.

\section{SUMMARY}

Alcohol use disorders are the most frequent addictions and is an important subject in clinical toxicology. Several abnormalities are associated with excessive alcohol consumption. They are useful in the diagnosis of alcohol abuse or dependence, especially during the follow-up of various treatment programs. The biological markers mostly used for diagnosis of alcohol use disorders are presented. Especially, gammaglutamyl transferase, aspartate and alanine aminotransferase, mean cell volume, carbohydrate-deficient transferrin, $\gamma$ CDT. No laboratory test exists that is reliable enough for the diagnosis of alcoholism. The combination of physician interview, questionnaires and laboratory markers is necessary for the diagnosis of alcohol use disorders.

\section{KEY-WORDS}

Alcoholism, gamma-glutamyl transferase, carbohydratedeficient transferrin $(C D T), \gamma-C D T$. 


\section{Introduction}

La détection de l'usage à risque, de l'usage nocif et de la dépendance à l'alcool est probablement encore largement sous-estimée par les professionnels de santé et pour certains auteurs (1), seuls 6 à $36 \%$ des personnes présentant un abus ou un syndrome de dépendance à l'alcool seraient correctement identifiés. L'intérêt de cette identification résulte des implications pratiques de celle-ci : repérage des personnes présentant un mésusage d'alcool avant l'apparition de complications irréversibles de cette alcoolisation (donc, dans l'idéal à l'étape de l'usage à risque et avant les complications) et identification de l'alcoolisation en tant que facteur étiologique dans certaines complications tant somatiques que psychiatriques. Ce facteur étiologique est sans doute largement sous-estimé dans ces dernières, notamment en cas de troubles anxieux, troubles de l'humeur ou troubles du sommeil, qui dans de nombreux cas prennent leur origine dans l'alcoolisation. L'entretien dirigé à la recherche de ces conduites d'alcoolisation est le plus souvent fructueux lorsqu'un climat de confiance est établi. Des questionnaires validés (type DETA ou AUDIT) $(1,2)$ permettent d'orienter vers le diagnostic d'usage ou de dépendance. Enfin les marqueurs biologiques d'alcoolisation seront utiles et pourront être utilisés, en tant qu'aide au dépistage de l'alcoolisation, qu'aide au dépistage des alcoolopathies, qu'outil de feedback motivationnel dans la prise en charge de l'abus ou de la dépendance ou qu'évaluation des stratégies thérapeutiques.

De nombreux marqueurs biologiques d'alcoolisation ont été utilisés, seuls ou en association. Cet article tente de faire le point sur les marqueurs biologiques actuels d'alcoolisation.

\section{Les marqueurs de 1'alcoo- lisme}

On note de très grandes différences dans l'apparition dans le temps des anomalies des marqueurs biologiques. La prise d'alcool peut être confirmée par le dosage de l'éthanol dans les liquides biologiques (sang, urine, air expiré, salive) par des méthodes enzymatiques ou chromatographiques (3). le dosage de l'acétate est aussi réalisable, quoique peu utilisé (4). Le 5hydroxytryptophol urinaire n'est pas de pratique courante (5). Tous ces marqueurs sont le reflet d'une consommation récente d'éthanol et ne peuvent donc être le reflet d'une consommation à long terme.

Les marqueurs biologiques d'une prise chronique d'alcool sont la gamma-glutamyl transférase $(\gamma \mathrm{GT})$, les transaminases, la transferrine déficiente en hydrates de carbone (CDT), la transferrine désialylée (SDT), le volume globulaire moyen (VGM).

\section{La gamma glutamyltransférase}

La gamma glutamyltransférase ( $\gamma \mathrm{GT}$ ) est une glycoprotéine localisée dans les membranes des cellules endothéliales, cette enzyme intervient dans le transport des peptides et le métabolisme du glutathion. Après consommation chronique d'alcool, les concentrations sériques de $\gamma \mathrm{GT}$ sont souvent élevées et cette augmentation serait due à une synthèse accrue de $\gamma G T$ et/ou une libération rapide de cette enzyme des hépatocytes endommagés ou nécrosés. Une variété de kits commerciaux employant des méthodes colorimétriques en cinétique ou en point final sont utilisables pour mesurer cette enzyme. Puisque l'usage excessif d'alcool est une des causes d'altération directe du foie, la

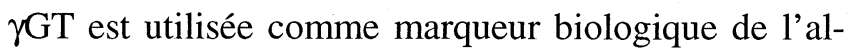
coolisme. Cependant, d'autres états pathologiques, tels que l'obésité, le tabagisme, les hépatopathies non liées à l'alcool, l'hypertension artérielle, l'hypertriglycéridémie, le diabète et quelques traitements médicamenteux

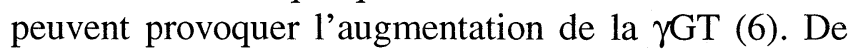
plus, beaucoup de sujets alcooliques ne souffrent pas d'hépatite et donc les concentrations sériques de $\gamma \mathrm{GT}$ restent dans les valeurs normales. Enfin, la fréquence, la durée et le profil de l'alcoolisation requis pour provoquer une augmentation des concentrations de $\gamma \mathrm{GT}$, n'ont pas encore été bien établis. La sensibilité de ce paramètre, comme marqueur varie selon les populations étudiées et la $\gamma \mathrm{GT}$ a une demi-vie comprise entre 14 et 26 jours. Cependant, la $\gamma \mathrm{GT}$ est le marqueur le plus utilisé, la sensibilité, selon les études est comprise entre 34 et $85 \%$, mais ce marqueur a une faible spécificité (7). Ce paramètre n'est donc pas le meilleur marqueur pour estimer une rechute au cours d'un traitement de sevrage. Les concentrations sériques de jGT peuvent ne pas retourner à la normale dans les quatre à cinq semaines après l'arrêt de l'alcool.

\section{L'aspartate aminotransférase (ASAT)}

Cette enzyme catalyse le transfert réversible d'un groupement aminé de l'acide aspartique à l'acide alphacétoglutarique pour former l'acide glutamique et l'acide oxaloacétique. Elle est présente dans la plupart des cellules des eucaryotes et participe au shunt acide malique-acide aspartique. Un taux élevé d'ASAT montre une altération hépatique plutôt qu'une consommation excessive d'alcool (7).

\section{L'alanine aminotransférase (ALAT)}

Cette enzyme est trouvée presque exclusivement dans le cytoplasme hépatique et est impliquée dans le métabolisme des acides aminés. L'ALAT peut être libérée 
dans le sang comme la conséquence d'une augmentation de la perméabilité membranaire ou d'une nécrose membranaire, secondaire à une altération des hépatocytes (7).

Les deux enzymes ASAT et ALAT ont été utilisées comme marqueur biologique d'une alcoolisation chronique. Les concentrations sériques de ces enzymes augmentent dans ces conditions, probablement comme le résultat d'une altération induite par l'alcool ou de la cytolyse hépatique. Ces enzymes, comme la $\gamma$ GT ne sont pas assez sensibles pour détecter la totalité des problèmes liés à l'alcool. Cependant, il a été montré que le rapport ASAT/ALAT peut être utilisé pour distinguer une hépatopathie liée à l'alcool d'une autre hépatopathie. Comme la valeur de l'ASAT est souvent plus grande que le valeur de l'ALAT dans les hépatopathies alcooliques, un rapport supérieur à 2 suggère que l'alcool pourrait être à l'origine de l'hépatopathie (8). Des kits commerciaux sont utilisés pour déterminer la valeur de ces enzymes et sont basés sur la détermination colorimétrique ou ultraviolette du produit de la réaction, la formation de celui ci étant proportionnelle à l'activité de l'enzyme.

\section{Le volume globulaire moyen (VGM)}

La consommation chronique d'alcool peut augmenter la taille des globules rouges. Le paramètre biologique correspondant à cette augmentation de taille est le volume globulaire moyen (VGM), qui détermine la taille des globules rouges, exprimée comme le volume occupé par un seul globule rouge. Cette détermination se fait dans des compteurs à cellules basées sur des techniques de cytométrie de flux, mesurant facilement et de manière peu coûteuse le VGM, il peut aussi être calculé. La cause la plus fréquente de l'augmentation du VGM est l'alcoolisation, et cette augmentation apparaît comme le résultat de l'action directe de l'alcool sur le développement des érythroblastes. Toutefois, cette augmentation peut aussi apparaitre dans des pathologies hépatiques non liées à l'alcool, des hémolyses, des myélodysplasies, des déficiences en folates et en vitamines B12, dans l'hypothyroïdie, des chimiothérapies ou d'autres thérapeutiques. La normalisation du VGM s'effectue en 40 jours et donc, n'est pas très sensible aux changements significatifs de la conduite d'alcoolisation (9). Cependant bien que ce paramètre ait une sensibilité faible, comme seul marqueur biologique de l'alcoolisme, il a une valeur accrue, quand il est associé à d'autres marqueurs (9).

\section{La transferrine déficiente en hydrates de carbone (CDT) et la transferrine désialy- lée (SDT)}

En 1976, Stibler (10) observait dans le liquide céphalo- rachidien et dans le sérum de sujets alcooliques une bande protéique anormale (point isoélectrique, pI : 5,7 ), identifiée comme étant une fraction de transferrine partiellement désialylée. Un lien est alors mis en évidence entre cette protéine et la consommation d'alcool. Des études structurales ont alors montré un déficit de la glycosylation de la transferrine. La transferrine est une bêtaglycoprotéine de 80000 daltons présentant plusieurs niveaux d'hétérogéneité. Le premier est dû à la substitution d'un ou plusieurs acides aminés au niveau de la chaîne polypeptidique de 679 acides aminés. On observe trois sous-types de transferrine : $\mathrm{C}(\mathrm{pI}$, $5,7)$ mis en évidence dans plus de $90 \%$ de la population caucasienne, B (pI plus faible) et D (pI, plus élevé). La partie $\mathrm{C}$ terminale de la molécule comprend deux chaines glycanniques. Elles peuvent être bi-, triou tétra-antennées, terminées par des molécules d'acide sialiques, dont le nombre dépend du degré de désialylation (en théorie de zéro à huit acides sialiques). Seules les fractions comportant au plus six acides sialiques sont observables. La forme dominante dans le sérum est la tétrasialotransferrine. Il existe deux sites de liaisons au fer, le contenu en fer participe également à l'héterogénéité de la transferrine. Il existe ainsi l'apotransferrine dépourvue d'atome de fer, les deux transferrines monoferriques ( $\mathrm{pI}$ identique) et la tansferrine diferrique.

La combinaison de ces hétérogénéités conduit à de nombreuses formes de transferrine. Seule l'hétérogénéité due à l'acide sialique est révélatrice de la consommation d'alcool. En effet, l'alcool ne semble pas affecter la synthèse de la chaîne polypeptidique, par contre la glycosylation est sous l'influence de l'alcool. L'anomalie principale observée est l'absence d'une chaîne glycannique, une anomalie de la biosynthèse des précurseurs de la $\mathrm{N}$-glycosylation en serait la cause, d'autres anomalies ont été décrites : les activités mannosyl transférase, galactosyl transférase et sialyl transférase hépatiques sont diminuées (11), de même que l'activité galactosyl transférase du sérum. L'acétaldéhyde issu du catabolisme de l'éthanol induit une diminution de l'activité galactosyl transférase (12). De plus, l'activité sialidase, en présence d'alcool augmente au niveau de la membrane de l'hépatocyte (11), ceci serait à l'origine d'une désialylation partielle de la transferrine avant sa sécrétion dans la circulation.

L'alcool est également impliqué dans le catabolisme de la transferrine. La désialylation est la première étape du catabolisme, l'activité des sialidases est augmentée chez les animaux intoxiqués à l'alcool.

La vitesse de la décroissance de la CDT après sevrage alcoolique montre une demi-vie d'environ 14 jours, identique à la CDT «normale» (13).La CDT peut être 
mise en évidence par plusieurs techniques :

- L'isoélectrofocalisation met en évidence et quantifie la CDT. Elle permet la caractérisation et l'étude des variants génétiques de la transferrine. Le résultat s'exprime en valeur absolue de disialotransferrine $(13,14)$, par le rapport des différentes fractions à la transferrine totale $(13,15)$ ou par le rapport de disialotransferrine/tétrasialotransferrine $(13,15)$.

- La chromatographie d'échange d'ions sur colonne permet de séparer les isoformes de la transferrine. Les résultats s'expriment en pourcentage de transferrine totale $(13,17)$ ou en unités pondérales $(13,18)$, mais les valeurs seuils dans ce cas ont été établies pour des sujets homozygotes C. Les variants B ont une CDT plus faible et les variants $\mathrm{D}$ une CDT augmentée avec cette méthode. Les variants D peuvent ainsi être considérés comme de faux négatifs (13). Ce résultat demande à être confirmé par une des deux techniques suivantes (19).

- La chromatographie liquide de haute performance permet d'identifier chaque fraction. Les résultats s'expriment en pourcentage de transferrine totale $(13,20)$ ou en valeur absolue (13). Elle permet ainsi de mettre en évidence les variants génétiques.

- L'électrophorèse capillaire peut aussi être utilisée en routine (21) pour mesurer la CDT.

- Des méthodes spécifiques, au moyen d'anticorps spécifiques permettent un dosage direct de la CDT. La CDT peut aussi être séparée avant dosage au moyen de lectines $(13,22)$.

L'isoélectrofocalisation quantifie généralement la disialotransferrine, les méthodes basées sur l'échange d'ions prennent en compte toutes les fractions contenant au plus deux acides sialiques, avec l'appoint de la trisialotransferrine, cette dernière forme doit être incluse, car la désialylation est un phénoméne progressif (23). Cette fraction augmente d'ailleurs chez les alcooliques. De plus, Keating et Coll. ont montré que la prise en compte de cette fraction augmente la sensibilité et la spécificité de la méthode (24). Les résultats s'expriment en valeur absolue $(\mathrm{mg} / \mathrm{L})$ ou en valeur relative (pourcentage de transferrine totale ou rapport CDT/ tétrasialotransferrine). Les valeurs varient selon les techniques entre 9 et $100 \mathrm{mg} / \mathrm{L}$ (valeur absolue) et entre 0,8 et $8,4 \%$ (valeur relative). Le mode d'expression des résultats ne semble pas modifier l'efficacité diagnostique de la CDT en l'absence d'autres pathologies.

En ce qui concerne, la sensibilité de la CDT, sa demivie assez courte peut conduire à des valeurs de sensibilité médiocre, lorsque le test est réalisé après plusieurs jours de sevrage. La CDT est plutôt le reflet de la consommation d'alcool des dernières semaines. Pour observer une élévation significative, il faut une consommation d'alcool prolongée (25).
Chez les alcooliques, en début de traitement, la sensibilité moyenne est de 0,72 contre 0,59 pour la $\gamma \mathrm{GT}$ (13). Avec des seuils de consommation de 24 à 80 $\mathrm{g} / \mathrm{jour}$, la $\gamma \mathrm{GT}$ et la CDT ont des sensibilités voisines peu élevées de l'ordre de 0,43 (13). La CDT apparaît plus sensible que la $\gamma$ GT chez le sujet jeune (26). La sensibilité de la CDT apparaît meilleure, à seuil de consommation alcoolique identique chez les sujets alcooliques, que chez les sujets abuseurs asymptomatiques. La CDT détecterait mieux les alcoolisations intermittentes et moins bien les consommations régulières que la YGT (27).

En ce qui concerne la spécificité, elle est en moyenne de 0,92 pour les sujets dont la consommation varie de 5 à $60 \mathrm{~g}$ par jour. Elle est supérieure à 0,85 quand le seuil de consommation est inférieur à $40 \mathrm{~g} /$ jour. La spécificité de la $\gamma \mathrm{GT}$ est de 0,87 , dans les mêmes populations.

La CDT n'est pas affectée par les pathologies suivantes : hypertension, bronchite, angine de poitrine, diabète, troubles du transit (spécificité :0,89) alors que

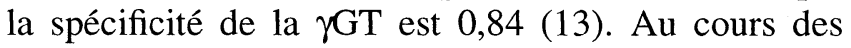
maladies hépatiques, la spécificité de la CDT reste élevée : 0,91 , alors que celle de la $\gamma \mathrm{GT}$ est basse : 0,36 (13). Il a également été montré que la variation de la valeur de la CDT, à la différence de celle de la $\gamma \mathrm{GT}$ est un bon témoin de la consommation d'alcool chez les sujets en bonne santé et chez les sujets alcooliques. La CDT détecte mieux les sujets à consommation irrégulière et le $\gamma \mathrm{GT}$, ceux à consommation régulière (consommation moyenne quotidienne de $80 \mathrm{~g}$ ). La décroissance de la CDT au cours du sevrage est observée chez tous les patients ayant une CDT initiale élevée et la majorité de ceux dont la valeur de CDT est inférieur à la valeur seuil (28). La normalisation s'effectue en 2 à 4 semaines (29).

\section{La transferrine désialylée}

De même, il a été montré que dans l'intoxication alcoolique, il était possible de mesurer la diminution en acide sialique de la transferrine ou sialic deficient transferrin (SDT). 50 à $80 \mathrm{~g}$ d'alcool par jour pendant au moins 8 jours se traduit par une production de mono-, di- ou trisialotransferrine regroupées sous le nom de SDT. A l'arrêt de l'absorption alcoolique, on assiste à une normalisation de la transferrine en 8 jours environ.

La mesure de la SDT est une technique directe, spécifique et rapide du degré de désialylation de la transferrine par voie immunoenzymatique au moyen d'une lectine spécifique. Les valeurs physiologiques sont comprises entre 52 et $77 \mathrm{mg}$ d'acide sialique par litre de sérum. Chez les buveurs modérés absorbant entre 20 et $50 \mathrm{~g}$ d'alcool par jour, on observe habituellement des taux compris entre 45 et $54 \mathrm{mg}$ d'acide sialique par litre de sérum. 
Chez les sujets qui consomment une quantité d'alcool supérieure à $60 \mathrm{~g}$ et plus d'alcool par jour, les taux sont abaissés à des valeurs de 15 à $45 \mathrm{mg}$ par litre de sérum. Chez un sujet naïf, absorbant $60 \mathrm{~g}$ d'alcool pendant 11 jours, on mesure le degré de désialylation à partir du 8ème jour. A l'arrêt complet de l'absorption alcoolique, la transferrine se normalise en 8 jours environ.

\section{Discussion}

L'ASAT, le rapport ASAT/ALAT mais aussi l'urée, les triglycérides, la bilirubine, les plaquettes et les monocytes conduisent à évoquer la prise d'alcool en cas d'anomalies. Un score composite a été proposé dans la détection des conduites d'alcoolisation (l'EDAC : early detection of alcohol consumption score) à partir d'examens biologiques usuels (30).

L'identification des sujets présentant des troubles liés à l'alcool par des marqueurs biologiques est un nouveau challenge méthodologique. Un nouveau marqueur doit avoir une sensibilité élevée. La méthode doit pouvoir s'appliquer facilement à la routine clinique. Les nombreuses recherches effectuées sur les marqueurs biologiques de l'alcoolisation montrent qu'il est utile d'en associer plusieurs pour détecter les sujets alcooliques (7).

Un modèle combinant cinq marqueurs a été proposé : ASAT, VGM, HDL-cholestérol/cholestérol total, triglycérides et le log des phosphatases alcalines. Ce modèle montre une sensibilité de $91 \%$ et une spécificité de $96 \%$, il permet de faire la différence entre les sujets présentant des troubles liés à l'alcool et les consommateurs occasionnels (31).

Shaper et coll ont proposé un modèle combinant $\gamma \mathrm{GT}$, HDL-cholestérol, acide urique, hémoglobine, et plomb, ce modèle avait une valeur discriminante de $41 \%$ pour les sujets présentant des troubles liés à l'alcool, comparé aux $17 \%$ pour le meilleur test isolé, la rGT (32).

Un modèle plus complexe inclut 10 paramètres : chlore, sodium, rapport bilirubine directe/totale, urée, HDL, monocytes, phosphore plaquette, ASAT et hémoglobine (34). Ce modèle permet de distinguer $98 \%$ des sujets ayant une forte consommation et $95 \%$ des sujets à consommation plus faible (33).

Bien que d'une utilité incontestable en tant d'aide au diagnostic et au suivi des personnes en difficulté avec l'alcool de nombreuses questions restent posées quant au marqueurs les plus couramment utilisés ( $\gamma$ GT et CDT et VGM). En ce qui concerne la spécificité, il semble que la CDT et le VGM soient les marqueurs les plus performants eu égard au peu de conditions autre que l'alcoolisation, susceptibles d'augmenter les valeurs. La spécificité de la YGT est plus faible en rai- son des nombreuses conditions susceptibles d'augmenter son taux.

En ce qui concerne la sensibilité il importe bien évidemment de se poser la question de la sensibilité par rapport à quoi. Bien évidemment CDT et $\gamma \mathrm{GT}$ ne sont pas des indicateurs d'un type de comportement (usage, usage à risque, abus ou dépendance) mais indique plus simplement l'existence d'une exposition à un xénobiotique. La littérature avance qu'une exposition de 50 à $80 \mathrm{~g} / \mathrm{j}$ durant 15 jours augmente le taux de CDT chez 81 à $94 \%$ des sujets. Pour la $\gamma$ GT une exposition supérieure à $80 \mathrm{~g} / \mathrm{j}$ pendant plusieurs semaines est nécessaire. On souligne néanmoins l'extrême variabilité individuelle de modification de ces marqueurs à l'exposition. Dans une population répondant aux critères diagnostiques de l'abus d'alcool le VGM avait une sensibilité de 0,24 et une spécificité de 0,96 , la $\gamma$ GT avait une sensibilité de 0,42 et une spécificité de 0,76 et la CDT avait une sensibilité de 0,67 et une spécificité de 0,97

Sillanaukee et Olsson (7) ont montré qu'il était préférable d'utiliser seulement deux marqueurs, la YGT et la CDT. Ils ont proposé à partir de plusieurs études de valider un modèle sur la base de ces paramètres. Ce modèle est appelé $\gamma$-CDT et est ainsi défini $\gamma$-CDT $=$ $0,8 \ln (\gamma \mathrm{GT})+1,3 \ln (\mathrm{CDT})$. Les sensibilités et spécificités moyennes de ce modèle étaient de $75 \%$ et $93 \%$ pour la $\gamma$-CDT, $58 \%$ et $94 \%$ pour la CDT, et $55 \%$ et $90 \%$ pour la $\gamma \mathrm{GT}$, en ce qui concerne les hommes. Elles étaient de $68 \%$ et $96 \%$ pour la $\gamma$-CDT, $40 \%$ et $94 \%$ pour la CDT et 52 et $96 \%$ pour la $\gamma \mathrm{GT}$, en ce qui concerne les femmes. Ce nouveau marqueur est donc simple à prendre en compte et différencie les sujets à forte consommation des sujets à consommation occasionnelle (7).

D'autres marqueurs ont également été décrits, comme les adduits à l'acétaldéhyde, les esters éthyliques des acides gras, les congénères, mais ils ne sont pas encore utilisés en routine(34).

D'autre part, l'analyse d'une autre matrice, comme les cheveux peut aussi permettre d'apprécier dans le temps les consommations en alcool, en mesurant l'éthylglucuronide ou encore les esters éthyliques des acides gras (35).

L'utilisation d'un marqueur biologique de l'alcoolisation nécessite en préalable de se poser la question de ce que l'on attend du dosage : dépistage d'une alcoolisation masquée potentiellement dangereuse pour l'individu et/ou la société ou outil de prise en charge en tant que feedback motivationnel et d'évaluation du soin. La proposition d'un dosage, le choix du marqueur, la fréquence des évaluations seront guidés par ce préalable indispensable. 


\section{Références}

1. Aertgeerts B., Buntinx F., Ansoms S., Fevery J. Screening properties of questionnaires and laboratory tests for the detection of alcohol abuse or dependance in a general practice population. Br. J. General Practice. 2001 ; 51 : 206-16.

2. Fiellin D.A., Reid M.C., O'Connor P.G. Screening for alcohol problems in primary care : a systematic. Rev. Arch Internh Med. 2000 ; 160 : 1977-89.

3. Deveaux M. Alcool éthylique : In : Kintz P. (coordinateur), Toxicologie et pharmacologie médicolégales, Option Bio, Elsevier, 1998 : 111-126.

4. Darvin A., Vion-Dury J., Viout P., Cozzone P.J. Rapid evaluation of ethanol content and metabolism in human plasma using quantitative proton magnetic resonance spectroscopy. Alcohol Alcohol, $1994 ; 29$ : 479-83.

5. Kroke A., Klipstein-Grobusch K., Hoffmann K., Terbeck I., Boeing H., Helander A. Comparison of self-reported alcohol intake with the urinary excretion of 5-hydroxytryptophol : 5 hydroxyindole-3-acetic acid, a biomarker of recent alcohol intake. Br. J. Nutr., $2001 ; 85$ : 621-7.

6. Whitfield J.B. Gamma glutamyl transferase. Crit. Rev. Clin. Lab. Sci., 2001 ; 38 : 263-355.

7. Sillanaukee P., Olsson U. Improved diagnostic classification of alcohol abusers by combining carbohydrate-deficient transferrin and g-glutamyl transferase. Clin. Chem., 2001 ; 47 : 681-5.

8. Allen J.P., Litten R.Z. The role of laboratory tests in alcoholism treatment. J. Subst. Abuse Treat. $2001 ; 20: 87-8$.

9. Sharpe P.C. Biochemical detection and monitoring of alcohol abuse and abstinence. Ann. Clin. Biochem. 2001 ; 38 : 652-4.

10. Stibler H., Allgulander C., Borg S., Kjellin K.G. Abnormal microheterogeneity of transferrin in serum and cerebrospinal fluid in alcoholism. Acta Med. Scand. $1978 ; 204$ : 49-56.

11. Xin Y., Lasker J.M., Lieber C.S. Serum carbohydrate-deficient transferrin : mechanism of increase after chronic alcohol intake. Hepatology. $1995 ; 22: 1462-8$.

12. Tuma D., Sorell M. Effect of ethanol on hepatic secretory proteins. Rec. Dev. Alcohol. 1984 ; 2 : 159-80.

13. Schellenberg F., Mouray $\mathrm{H}$. La transferrine déficiente en hydrates de carbone : quoi de neuf 20 ans plus tard ? Ann. Biol. Clin. 2000 ; 58 : 298-309.

14. Xin Y., Lasker J.M., Rosman A.S., Lieber C.S. Isoelectric focusing/western blotting : a novel and practical method for quantification of carbohydrate deficient transferrin in alcoholics. Alcohol Clin. Exp. Res. 1991 ; 15 : 814-21.

15. Bean P., Peter J.B. A new approach to quantitate carbohydratedeficient transferrin isoforms in alcohol abusers : partial iron saturation in isoelectric focusing/immunobloting and laser densitometry. Alcohol Clin. Exp. Res. 1993 ; 17 : 1163-70.

16. Godsell P.A., Whitfield J.B., Congrave K.M., Hanretty S.J., Saunders J.B. Carbohydrate deficient transferrin levels in hazardous alcohol consumption. Alcohol Alcohol. 1995 ; 30 :61-6.

17. Vitala K., Lähdesmäki K., Niemalä O. Comparison of the Axis\%CDT TIA and the CDTect method as laboratory tests of alcohol abuse. Clin. Chem. 1998 ; 44 : 1209-15.

18. Schellemberg F., Martin M., Caces E., Bénard JY., Weill J. Nephelemetric determination of carbohydrate deficient transferrin. Clin. Chem. 1996 ; 42 : 551-57.
19. Helander A., Eriksson G., Stibler H., Jeppsson J.O. Interference of transferrin isoform types with carbohydratedeficient transferrin quantification in the identification of alcohol abuse. Clin. Chem. 2001 ; 47 : 1225-33.

20. Sillanaukee P., Löf K., Härlin A., Martensson O., Brandt R., Seppä K. Comparison of different methods for detecting carbohydrate-deficient transferrin. Alcohol Clin. Exp. Res. 1994 ; $18: 1150-5$.

21. Tagliaro F., Crivellente F., Manetto G., Puppi I., Deyl Z., Marigo M. Optimized determination of carbohydrate-deficient transferrin isoforms in serum by capillary zone electrophoresis. Electrophoresis $1998 ; 19: 3033-9$.

22. Yoskitawa K., Umetsu K., Shinzawa H. et al. Determination of carbohydrate-deficient transferrin separated by lectin affinity chromatography for detecting chronic alcohol abuse. FEBS Lett. $1999 ; 458: 112-6$.

23. Heggli DE., Aurebekk A., Granum B., Westby C., Sundrehagen E. Should trishalotransferrin be included when calculating CDT for diagnosing elevated alcohol intake ? Alcohol Alcohol. $1996 ; 31$ : 381-4.

24. Keating J., Cheung C., Peters T.J., Sherwood R.A. Carbohydrate-deficient transferrin in the assesement of alcohol misuse : absolute or relative measurements ? A comparison of two methods with regard to total transferrin concentration. Clin. Chim. Acta $1998 ; 272: 159-69$.

25. Huseby N.E., Nilsson O., Kanitz R.D. Evaluation of two biological markers combined as a parameter of alcohol dependency. Alcohol Alcohol. 1997 ; 32 : 731-7.

26. Sillanaukee P., Aalto M., Seppa K. Carbohydrate-deficient transferrin and conventional alcohol markers, as indicators for brief intervention among heavy drinkers in primary health care. Alcohol Clin. Exp. Res. 1998 ; 22 : 892-6.

27. Schellenberg F., Bénard J.Y., Cacés E., Weill J. Influence de la quantité d'alcool et de la régularité de sa consommation sur l'élévation de la transferrine désialylée sérique. Cahiers de l'Ireb. 1989 ; 9 : 195-201.

28. Planche F., Hourcade F., Albuisson E., Meunier M.N., Planche R., Reynaud M. Dosage de la carbohydrate deficient transferrin : diagnostic des patients alcooliques dépendants à gammaGT normales. Alcoologie. 1997 ; 19 : 407-12.

29. Sillanaukee P, Ponnio M, Seppa K. Sialic acid : new potential marker of alcohol abuse. Alcohol Clin. Exp. Res. (1999) $23: 1039-43$.

30. Bean P., Harasymiw J., Peterson C.M., Javors M. Innovative technologies for the diagnosis of alcohol abuse and monitoring abstinence. Alcohol Clin. Exp. Res. 2001 ; 25 (2) : 309-16.

31. Sillanaukee P. The diagnosic value of a discriminant score in the detection of alcohol abuse. Arch. Path. Lab. Med. 1992 ; 924-9.

32. Sharper A.G., Pocock S.J., Ashby D., Walker M., Whitehead T.P. Biochemical and haematological response to alcohol intake. Ann. Clin. Biochem. $1985 ; 22$ : 50-61.

33. Hartz A., Guse C., Kajdacsy-Balla A. Identification of heavy drinkers using a combination of laboratory tests. J. Clin. Epidemiol. $1997 ; 50$ : 1357-68.

34. Musshoff F., Daldrup T. Determination of biological markers for alcohol abuse. J. Chromatogr., 1998 ; 713 : 245-64.

35. Pragst F., Spiegel K., Sporkert F., Bohnenkamp M. Are there possibilities for the detection of chronically elevated alcohol consumption by hair analysis ? a report about the state of investigation. Forensic Sci., $2000 ; 107$ : 201-23. 